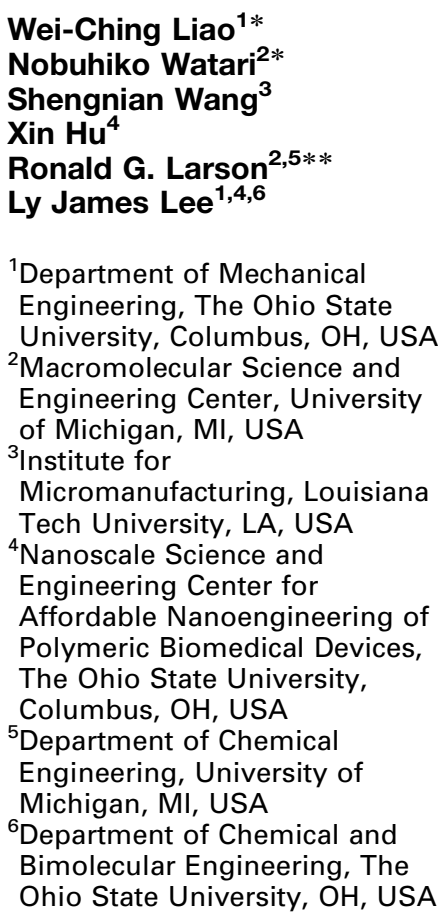

Received February 14, 2010

Revised May 11, 2010

Accepted May 14, 2010

\section{Research Article \\ Conformation dependence of DNA electrophoretic mobility in a converging channel}

\begin{abstract}
The electrophoresis of $\lambda$-DNA is observed in a microscale converging channel where the center-of-masses trajectories of DNA molecules are tracked to measure instantaneous electrophoretic (EP) mobilities of DNA molecules of various stretch lengths and conformations. Contrary to the usual assumption that DNA mobility is a constant, independent of field and DNA length in free solution, we find DNA EP mobility varies along the axis in the contracting geometry. We correlate this mobility variation with the local stretch and conformational changes of the DNA, which are induced by the electric field gradient produced by the contraction. A "shish-kebab" model of a rigid polymer segment is developed, which consists of aligned spheres acting as charge and drag centers. The EP mobility of the shish-kebab is obtained by determining the electrohydrodynamic interactions of aligned spheres driven by the electric field. Multiple shishkebabs are then connected end-to-end to form a freely jointed chain model for a flexible DNA chain. DNA EP mobility is finally obtained as an ensemble average over the shishkebab orientations that are biased to match the overall stretch of the DNA chain. Using physically reasonable parameters, the model agrees well with experimental results for the dependence of EP mobility on stretch and conformation. We find that the magnitude of the EP mobility increases with DNA stretch, and that this increase is more pronounced for folded conformations.
\end{abstract}

\section{Keywords:}

Conformation-dependent mobilities / DNA / Electrophoretic mobility / Free solution mobility / Microfluidic converging channel DOI 10.1002/elps.201000081

\section{Introduction}

Electrophoresis is the migration of charged species in a medium under an applied electric field. This phenomenon has been widely utilized in electrokinetics-related research and applications such as gel electrophoresis separation [1], study of polyelectrolyte dynamics in electrokinetic flows $[2,3]$, and in electroporation for gene delivery [4]. DNA is a primary material used in many of these applications because of its important role in both biological science and single macromolecule dynamics. In gel electrophoresis, DNA separation is achieved due to the dependence of

Correspondence: Professor Ly James Lee, Department of Chemical and Biomolecular Engineering, The Ohio State University, OH 43210, USA

E-mail: leelj@chbmeng.ohio-state.edu

Fax: +1-614-292-8685

Abbreviations: ELS, electrophoretic light scattering; EP, electrophoretic mobility on molecular size of DNA molecules in the gel. In recent years, many microfluidic devices have been designed to separate DNA molecules by taking advantage of the similarity between the size scales typical in microfluidic geometries and the size of the DNA molecule itself. Examples of such devices include micro-obstacle arrays [5], entropy traps [6], and molecular ratchets [7].

Electrophoretic (EP) mobility $\left(\mu_{\mathrm{EP}}\right)$, defined as the migration velocity obtained under a unit electric field strength $\left(\mu_{\mathrm{EP}}=U_{\mathrm{EP}} / E\right)$, is usually set by the size, shape, and charge density of charged species and the ionic strength and viscosity of the medium. In general, electrohydrodynamic interactions are strongly screened in the electrophoresis of dilute polyelectrolytes because of the movement of surrounding counterions [8]. However, as shown by Long and Ajdari, screening of electrohydrodynamic interactions is not complete, and despite the presence of mobile

\footnotetext{
*These authors contributed equally to this work

**Additional corresponding author: Dr. Ronald G. Larson

E-mail: rlarson@umich.edu
} 
counterions there should remain a rather steep power-law decay of electrohydrodynamic microflows near the DNA [9]. Transverse migration has also been found in simulations [10] and experimental observations [11, 12] of DNA electromigration in thin channels, which again shows the importance of electrohydrodynamic interactions.

There are several ways to measure the EP mobility of DNA, including the use of CZE [13], electrophoretic light scattering (ELS) [14], and optical velocimetry. These approaches all rely on the relation $\mu_{\mathrm{EP}}=\Delta d / E \Delta t$, where $E$ is the local electric field strength, and $\Delta d$ the displacement of charged species within a time interval of $\Delta t$. In CZE, $\Delta d$ is the distance between the injection and detection locations $(>10 \mathrm{~cm})$ and the traveling time $(\Delta t)$ is determined by the UV signal carried by the DNA molecules. ELS determines $\Delta d$ and $\Delta t$ by measuring the Doppler shift of light scattered from moving DNA molecules. Both CZE and ELS provide an average value of EP mobility from multiple DNA molecules. The recently developed microscale particle tracking velocimetry ( $\mu \mathrm{PTV})[15]$ can obtain the instantaneous velocity of a single charged particle. Observation of the dynamics of individual, fluorescently-stained DNA molecules enables the identification of conformations taken on by DNA molecules during the coil-stretch transition. By combining $\mu$ PTV with single-molecule DNA measurements, we will here obtain both the velocity and conformation of individual DNA molecules in a well-defined electric field.

In free-solution electrophoresis (i.e. with no gel or other barrier to DNA motion), DNA molecules mostly remain in their coiled conformation and the EP mobility usually remains constant during migration. However, in cross-slot and converging geometries, due to the gradient in field strength, the DNA molecule can undergo a coil-stretch transition [16]. Here, we investigate the impact of DNA conformational change on EP mobility. To do so, we use a microscale converging channel to create an electric field gradient that stretches DNA molecules and investigate whether or not this stretch induces a change in the EP mobility. Because of "molecular individualism" [17], we can observe multiple DNA conformations in a single experiment and the time dependence of the conformation as it traverses the geometry. We will calculate the instantaneous DNA EP mobility of each molecule and correlate this with its instantaneous conformation. A theoretical analysis will also be employed to estimate the center-of-mass EP migration of DNA, including the effects of electrohydrodynamic interactions alluded to above.

\section{Materials and methods}

\subsection{Experimental observation and measurement}

\subsubsection{Fabrication of the converging channel}

To study the dependence of EP mobility on the DNA conformation in free solution, a microscale converging channel was designed and fabricated on a PMMA plate. The dimensions of the channel are shown in Fig. $1 \mathrm{~A}$, where $L, W$, $l, w_{1}$, and $w_{2}$ are $1.5 \mathrm{~cm}, 300 \mu \mathrm{m}, 300 \mu \mathrm{m}, 20 \mu \mathrm{m}$, and $130 \mu \mathrm{m}$, respectively. Upon application of a DC voltage across the channel, the magnitude of the electric field $\mathbf{E}$ increases in the converging section because of the tapered contraction in the cross-section area. The increased electric field can stretch and consequently change DNA conformation when DNA migrates through the converging channel. The PMMA microfluidic chip was fabricated first by patterning the microfluidic structure on the silicon wafer using photolithography. A negative photoresist, SU-8 50 (Microchem) was used to fabricate the female mold of the channel. A PDMS male mold was then made by casting the mixture of 10:1 ratio (by weight) of PDMS precursor and curing agent (Sylgard 184, Dow Corning) on the patterned SU-8 structure using soft lithography. The mixture was degassed in vacuum for an hour and then cured overnight at $65^{\circ} \mathrm{C}$ in an oven. The patterned PDMS mold was thus the negative replica of the microscale converging channel. The PMMA converging channel was then fabricated by using the PDMS as the soft mold in the hot embossing process. In the hot embossing, the PDMS mold and the PMMA plate were sandwiched between two glass plates in a heating press (Carver IN) at $180^{\circ} \mathrm{C}$. The inlet and outlet of the channel were created by drilling holes with
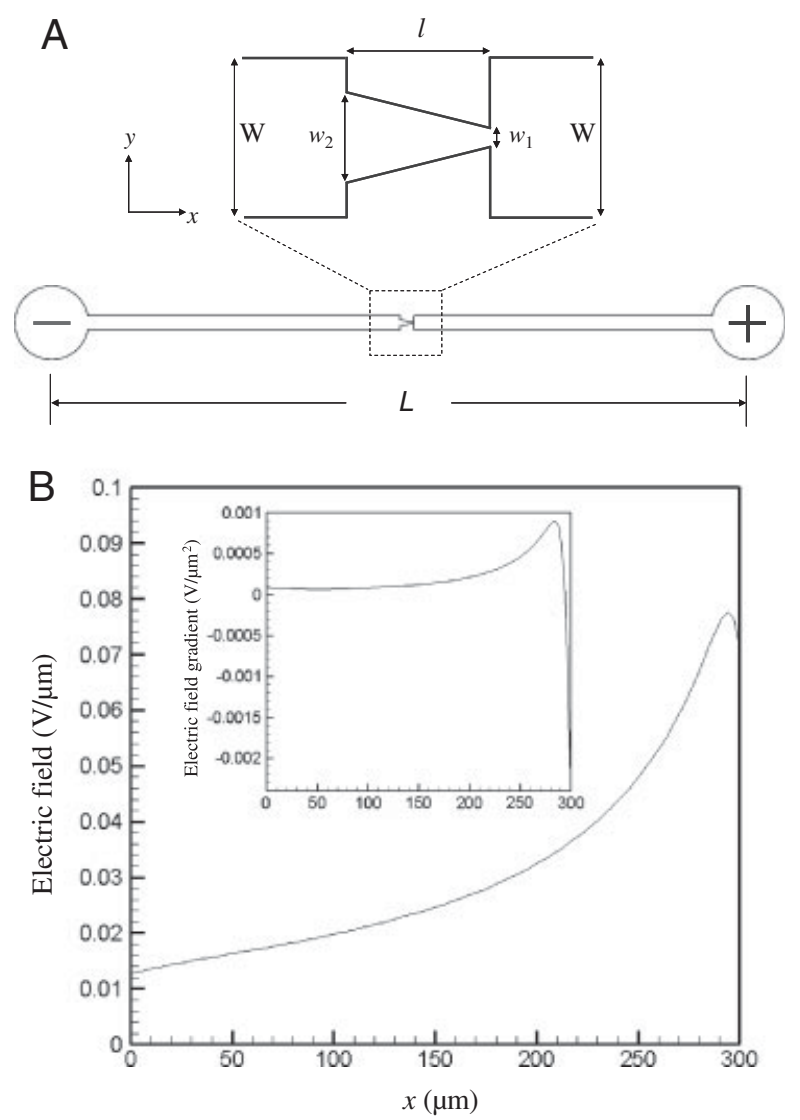

Figure 1. (A) Schematic diagram of the microscale converging channel. (B) The calculated electric field and electric field gradient along the converging channel central line. 
diameter of $1.6 \mathrm{~mm}$. Finally, a $50 \mu \mathrm{m}$ thick PMMA film was thermally laminated onto the surface of the microfluidic plate to form the closed channel.

\subsubsection{Electrophoresis setup}

$\lambda$-DNA (48.5 kbps, New England Biolab) is used to study DNA EP mobility. A dilute solution $(\sim 0.03 \mu \mathrm{g} / \mathrm{mL})$ of $\lambda$-DNA in a $10 \mathrm{mM}$ tris- $\mathrm{HCl}, 2 \mathrm{mM}$ EDTA, and $10 \mathrm{mM}$ $\mathrm{NaCl}$ buffer at $p \mathrm{H} 8.0$ was labeled with fluorescent YOYO-1 dye at a dye-to-base-pair molar ratio of 1:5. Under these conditions, $\lambda$-DNA attains a contour length of about $21 \mu \mathrm{m}$ when fully stretched [18]. To increase the viscous drag, the bulk viscosity of DNA solution was adjusted by adding glucose and sucrose. In this work, $18 \% \mathrm{w} / \mathrm{w}$ glucose and $40 \% \mathrm{w} / \mathrm{w}$ sucrose were added so that the final bulk viscosity of DNA solution is $30 \mathrm{cp}$. To study the EP migration of DNA molecules in the converging channel, the whole chip was first mounted onto the stage of an inverted epi-fluorescence microscope (TE 2000S, Nikon, Japan). Then, the DNA solution was pumped to fill to channel from the inlet. Electrodes were placed in the wells of inlet and outlet with applied voltage of $100 \mathrm{~V}$ applied along the $1.5 \mathrm{~cm}$ long microchannel. An electric field $\mathbf{E}$ was generated along the converging channel, which gives a high enough field gradient to induce conformational changes in the DNA molecules. The dynamics of DNA were observed with a $100 x / 1.3$ NA oil immersion objective lens and recorded by a Coolsnap HQ CCD camera (Roper Scientific, Tempe, AZ). Single DNA dynamics were studied recently by Brownian dynamics simulations and compared with experimental results [16]. For simplicity, a constant DNA EP mobility was used in the previous simulations. Our goal here, however, is to track each DNA molecule and determine the relationship between EP mobility and DNA conformation.

\subsubsection{Measurement of EP mobility}

The EP mobility is determined by applying the finite difference scheme to the trajectory of the DNA center-ofmass. From the measured distance traveled by the DNA between two video frames, the time interval between the frames, and the electric field strength computed by the finite element method [16] as shown in Fig. 1B, a transient mobility can easily be calculated. The EP mobility obtained in this way is an "instantaneous EP mobility" that changes as the DNA molecule migrates. Within the time interval between frames of $0.052 \mathrm{~s}$, DNA molecules travel a distance of $5 \pm 2 \mu \mathrm{m}$. Over this distance, the electric field gradient varies by only a few percent and can be taken to be constant. Also, the electric field varies at most by around $18 \%$ over the length of the DNA molecule, and so the field at the molecule's center is used to compute the instantaneous mobility. As mentioned in our early work, the deformation of DNA molecules under an extensional electric field is similar to that under a hydrodynamic extensional flow. The angle that the stretched DNA molecule makes with respect to the axis of the device (the $x$ direction) is zero along the center line of the channel and is small in the contraction zone away from the center line [16]. Thus, only DNA located near the central line is chosen for investigation so that DNA is stretched mainly along the $x$-direction with minimal wall effects. It can be seen in Fig. 1B that the electric field strength develops steep gradient near the end of channel. DNA chain tumbling was observed in this region. Therefore, the tracking of DNA was stopped when the DNA arrived close to the small end $(x=280 \mu \mathrm{m})$ of the contraction, before the region of steep gradients. The contribution of center-of-mass diffusion to the noise in the distance traveled over the time interval between viewing frames can be estimated from the DNA diffusion coefficients measured by Smith et al. [19]. The root mean square displacement of $\lambda$-DNA is thereby determined to be about $0.04 \mu \mathrm{m}$ over the 0.052 time interval, which is 100 times smaller then the average distance traveled by the DNA molecule over that time interval. Therefore, the contribution of Brownian diffusion to the noise in the measurement of EP mobility is negligible. In total, 32 DNA molecules were traced. The number of captured frames per DNA molecule varied from 9 to 27 depending on the migration speed in the contraction zone. A total of 340 EP mobility data points were obtained in this study.

\subsection{Theoretical modeling}

\subsubsection{A shish-kebab model}

The EP mobility of a charged spherical particle $\mu_{0}$ is determined by its radius $a$, carried charge $Q$, the Debye length $\kappa^{-1}$ and the viscosity of the solvent $\eta$ (i.e. $\mu_{0}=\mu_{0}$ $(a, Q, \kappa, \eta))[20]$, and the "far-field" flow perturbation induced by the electrophoresis of a charged spherical particle decays with distance from the particle $r$ as $\sim 1 / r^{3}$ [9], i.e. the velocity perturbation at position $\mathbf{r}_{i}$ that results from the EP motion of

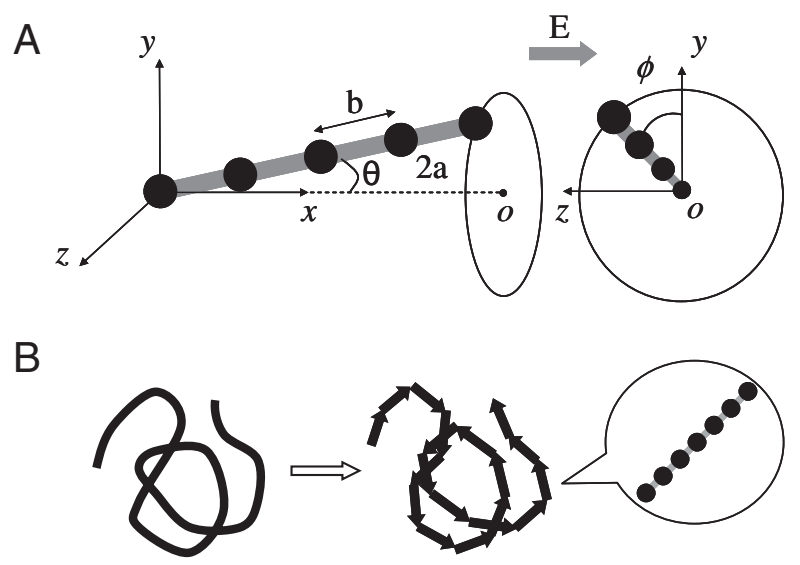

Figure 2. (A) Schematic diagram of shish-kebab model. (B) A freely jointed chain consisting of multiple charged "shishkebab" segments. 
a charged particle at $\mathbf{r}_{j}$ is

$$
\begin{array}{r}
\mathbf{v}^{\mathrm{EPHI}}\left(\mathbf{r}_{i}, \mathbf{r}_{j}\right)=\mathbf{S}\left(\mathbf{r}_{i j}\right) \cdot \mathbf{E}\left(\mathbf{r}_{j}\right), \\
\mathbf{S}\left(\mathbf{r}_{i j}\right)=\frac{Q}{4 \pi \eta \kappa^{2} r_{i j}^{3}}\left(3 \frac{\mathbf{r}_{i j} \mathbf{r}_{i j}}{r_{i j}^{2}}-\mathbf{I}\right),
\end{array}
$$

where $\mathbf{I}$ is the unit tensor, and $\mathbf{r}_{i j}=\mathbf{r}_{i}-\mathbf{r}_{j}$.

We propose a simple "shish-kebab" model for the EP mobility of a short, rigid segment of a longer, semi-flexible, charged, polymer chain. We generate a shish-kebab by connecting charged spherical particles with thin rods on a straight line as shown in Fig. 2A. Assuming that each particle has charge $Q$ and radius $a$, and that the distance between two adjacent particles is $b$, the center-of-mass velocity of a shish-kebab, which consists of $N_{S K}$ spheres in the electric field $\mathbf{E}=(E, 0,0)^{T}$ can be expressed as the following for $N_{\mathrm{SK}} \gg 1$ :

$$
\begin{aligned}
\mathbf{v}^{\mathrm{SK}}= & \mu_{0} \mathbf{E}+\frac{1}{N} \sum_{i=1}^{N_{\mathrm{SK}}} \sum_{j \neq i} \mathbf{v}^{\mathrm{EPHI}}\left(\mathbf{r}_{i}, \mathbf{r}_{j}\right) \\
= & \mu_{0} \mathbf{E}+\frac{1}{N} \sum_{i=1}^{N_{\mathrm{SK}}} \sum_{j \neq i} \frac{Q E}{2 \pi \eta \kappa^{2}(b|i-j|)^{3}}\left(\begin{array}{c}
3 \cos ^{2} \theta-1 \\
3 \cos \theta \sin \theta \cos \phi \\
3 \cos \theta \sin \theta \sin \phi
\end{array}\right) \\
= & \mu_{0} \mathbf{E}+\frac{1}{N} \frac{Q E}{\pi \eta \kappa^{2} b^{3}} \\
& \sum_{n=1}^{N_{\mathrm{SK}}}\left(\frac{1}{1^{3}}+\frac{1}{2^{3}}+\cdots+\frac{1}{n^{3}}\right)\left(\begin{array}{c}
3 \cos ^{2} \theta-1 \\
3 \cos \theta \sin \theta \cos \phi \\
3 \cos \theta \sin \theta \sin \phi
\end{array}\right) \\
\cong & \mu_{0} \mathbf{E}+\frac{1.2 Q E}{\pi \eta \kappa^{2} b^{3}}\left(\begin{array}{c}
3 \cos \theta-1 \\
3 \cos \theta \sin \theta \cos \phi \\
3 \cos \theta \sin \theta \sin \phi
\end{array}\right) \\
= & \mu_{0} \mathbf{E}+\mu_{1} E\left(\begin{array}{c}
3 \cos \theta-1 \\
3 \cos \theta \sin \theta \cos \phi \\
3 \cos \theta \sin \theta \sin \phi
\end{array}\right)
\end{aligned}
$$

where $\theta$ and $\varphi$ are angles describing the orientation of the shish-kebab as defined in Fig. 2A, $\mu_{1}$ is defined as $\mu_{1} \equiv 1.2 Q E / \pi \eta \kappa^{2} b^{3}$, and the approximation from the third line to the fourth is correct within $4 \%$ error for $N_{\mathrm{SK}}>10$. From the second term of Eq. (2), the EP velocity of the center-of-mass in the direction of the electric field is highest: $\left(\mu_{0}+2 \mu_{1}\right) E$ at $\theta=n \pi$, and smallest: $\left(\mu_{0}-\mu_{1}\right)$ E at $\theta=\pi(2 n+$ $1) / 2$, where $n$ is an integer. Moreover, a migration velocity component perpendicular to the electric field is induced when $\theta \neq n \pi / 2$. These results qualitatively agree with theoretical and experimental studies on the electrophoresis of spheroidal particles [21] and charged polymers under deformation in the Poiseuille flow [10]. Also, according to Ref. [21], the ratio of the EP mobilities of an infinite cylinder oriented parallel to the field, perpendicular to the field, and a sphere of the same surface charge density with the same radius as the cylinder is $1: 1 / 2: 2 / 3$ in the regime $\kappa a \leq 1$ where $a$ is the radius of a cylinder or a sphere, while this ratio converges to $1: 1: 1$ when $\kappa a$ is large $\left(\sim 10^{2}\right)$. By comparing this ratio in the regime $\kappa a \leq 1$ with our result in Eq. (2) for a shish-kebab, i.e. $1: 1 / 2: 2 / 3=\left(\mu_{0}+2 \mu_{1}\right):\left(\mu_{0}-\mu_{1}\right): \mu_{0}$, we find the relation: $\mu_{1}=\mu_{0} / 4 \quad(\kappa a \leq 1)$. In the regime $\kappa a>1, \mu_{1}$ decreases from $\mu_{0} / 4$ as $\kappa a$ increases. Our experimental condition satisfies $\kappa a \leq 1$ with $a$ being the local radius of DNA chain, which allows us to take $\mu_{1}=\mu_{0} / 4$.

\subsubsection{Model for a flexible molecule}

To estimate the EP velocity of a charged polymer, we connect together shish-kebabs, each of length equal to the Kuhn step length of the polymer chain, to assemble a flexible sub-chain, just as rods are connected to form a freely jointed chain, as shown in Fig. 2B. The freely jointed subchain is considered to be a single "spring" representing a portion of the whole chain. Each sub-chain, or spring, contains enough shish-kebabs to form a micro-canonical "ensemble" of shish-kebabs over which averages can be taken, but is a small enough portion of the whole chain that each shish-kebab within that spring has behavior that is, on average, identical to that of any other shish-kebab in the same spring. That is, variations in electric field gradient and stretching force are negligible over the small size of the subchain represented by a single spring. This is the standard approximation used in bead-spring modeling of polymer chains. Therefore, we model a chain with four hierarchical levels, which from the smallest to the largest are: (i) spheres, (ii) shish-kebab, (iii) sub-chain, and (iv) chain. In the above Section, Eq. (2) describes level 2 (shish-kebab) of the hierarchy, deriving the mobility at this level from the electrokinetics of level 1, given by Eq. (1).

We now extend our model to level 3, that of a sub-chain composed of many connected shish-kebabs. When disconnected from each other, the shish-kebabs have differing velocities that depend on the orientation angles of the individual shish-kebabs. When connected, forces are exerted between shish-kebabs that equalize their migration speeds. This connector force exerted on one shish-kebab to bring its speed up (or down) to that of the whole sub-chain is countered by an equal and opposite connector force that this shish-kebab exerts on the rest of the sub-chain, bringing the velocity of the rest of the sub-chain down (or up) to that of the one shish-kebab. Since the translational drag coefficient of a thin filament moving orthogonal to its axis is roughly twice that of one moving parallel to its axis [22], this effect can easily be accounted for by including a weighting factor that is calculated using this drag coefficient in the averaging of the velocities of the isolated shish-kebabs. The ensembleaveraged center-of-mass mobility of the sub-chain can be evaluated as follows. Since each shish-kebab, say shishkebab $i$, in a sub-chain, if isolated from the others, would have an orientation-dependent EP velocity which would differ from the velocity of the other shish-kebabs in the subchain, there must be a connector force $\mathbf{f}_{i}$ on shish-kebab $i$, which forces it to move at the center-of-mass velocity $\mathrm{V}$ of rest of the sub-chain. This connector force is just the drag force required to change the velocity of the shish-kebab from 
its velocity when isolated, namely, $\mathbf{v}_{i}^{\mathrm{SK}}$, to the velocity of the rest of the sub-chain, namely, $\mathbf{V}$. This drag force $\mathbf{f}_{i}$ is therefore proportional to $\mathbf{v}_{i}^{\mathrm{SK}}-\mathbf{V}$, and is dependent on the orientation of shish-kebab $i$. We can obtain it from the formula for the orientation-dependent drag force on a moving cylinder, which is given by [22]

$$
\begin{array}{r}
\mathbf{f}_{i}=\zeta_{\|} \mathbf{u}_{i} \mathbf{u}_{i} \cdot\left(\mathbf{v}_{i}^{\mathrm{SK}}-\mathbf{V}\right)+\zeta_{\perp}\left(\mathbf{I}-\mathbf{u}_{i} \mathbf{u}_{i}\right) \cdot\left(\mathbf{v}_{i}^{\mathrm{SK}}-\mathbf{V}\right) \\
=\left[\zeta_{\|} \mathbf{u}_{i} \mathbf{u}_{i}+\zeta_{\perp}\left(\mathbf{I}-\mathbf{u}_{i} \mathbf{u}_{i}\right)\right] \cdot\left(\mathbf{v}_{i}^{\mathrm{SK}}-\mathbf{V}\right)=\mathbf{Z}_{i} \cdot\left(\mathbf{v}_{i}^{\mathrm{SK}}-\mathbf{V}\right),
\end{array}
$$

where $\zeta_{\|}$and $\zeta_{\perp}$ are the drag coefficients of a shish-kebab parallel and perpendicular to the axis of the shish-kebab respectively, and satisfy $\zeta_{\perp}=2 \zeta_{\|}$. Here $\mathbf{u}_{i} \equiv\left(\cos \theta_{i}, \sin \theta_{i} \cos \right.$ $\left.\phi_{i}, \sin \theta_{i} \sin \phi_{i}\right)^{T}$ is the unit orientation vector of the shishkebab, and we define $\mathbf{Z}_{i} \equiv\left[\zeta_{\|} \mathbf{u}_{i} \mathbf{u}_{i}+\zeta_{\perp}\left(\mathbf{I}-\mathbf{u}_{i} \mathbf{u}_{i}\right)\right]$ to be the drag tensor of the shish-kebab. The term $\mathbf{u}_{i} \mathbf{u}_{i}$ can be explicitly written as:

$\mathbf{u}_{i} \mathbf{u}_{i}=$

$$
\left(\begin{array}{ccc}
\cos ^{2} \theta_{i} & \cos \theta_{i} \sin \theta_{i} \cos \phi_{i} & \cos \theta_{i} \sin \theta_{i} \sin \phi_{i} \\
\cos \theta_{i} \sin \theta_{i} \cos \phi_{i} & \sin ^{2} \theta_{i} \cos ^{2} \phi_{i} & \sin ^{2} \theta_{i} \cos \phi_{i} \sin \phi_{i} \\
\cos \theta_{i} \sin _{i} \sin \phi_{i} & \sin ^{2} \theta_{i} \cos \phi_{i} \sin \phi_{i} & \sin ^{2} \theta_{i} \sin ^{2} \phi_{i}
\end{array}\right) .
$$

Since these connector forces are all internal forces, they must balance each other out (by Newton's third law) and sum to zero: $\sum_{i=1}^{N_{K}} \mathbf{f}_{i}=0$, where $N_{K}$ is the number of Kuhn steps in a sub-chain. Therefore, we find

$\sum_{i=1}^{N_{K}} \mathbf{Z}_{i} \cdot \mathbf{V}=\sum_{i=1}^{N_{K}} \mathbf{Z}_{i} \cdot \mathbf{v}_{i}^{\text {SK }}$

Since each shish-kebab in the sub-chain sees the same orientation distribution, when $N_{K}$ is large, we can replace the summations in the above by $N_{K}$ times the ensemble average of the orientation of each shish-kebab, giving

$$
\begin{gathered}
N_{K}\langle\mathbf{Z}\rangle \cdot \mathbf{V}=N_{K}\left\langle\mathbf{Z} \cdot \mathbf{v}^{\mathrm{SK}}\right\rangle, \\
\quad \text { or }\langle\mathbf{Z}\rangle \cdot \mathbf{V}=\left\langle\mathbf{Z} \cdot \mathbf{v}^{\mathrm{SK}}\right\rangle,
\end{gathered}
$$

where we omit the index $i$ as a result of the ensemble averaging, $\langle A\rangle=\int_{0}^{2 \pi} \int_{0}^{\pi} P(\theta, \phi) A \sin \theta d \theta d \phi$, and $P(\theta, \phi)$ is the orientation probability distribution for a shish-kebab. Note here that we neglect the orientation-correlation between neighboring shish-kebabs caused by the bending elasticity of the chain. To obtain the ensemble averages, we now assume that this sub-chain is under a fixed stretching force $\mathrm{F}^{\text {str }}(\mathrm{s})$ that stretches the sub-chain to an average stretch ratio $\mathrm{s} / \mathrm{L}$ (L: maximum stretch) in the $\mathrm{x}$-direction (i.e. the field direction). As mentioned above, the electric field $\mathbf{E}$ is assumed to be spatially uniform over this sub-chain (or spring), although the field is varying over the length of the whole molecule, which is what leads to polymer stretching. The ensemble average of shishkebab angles can be calculated from a Boltzmann distribution:

$P(\theta, \phi)=\frac{1}{Z} \exp \left\{F^{\mathrm{str}}(s) L_{K} \cos \theta / k_{B} T\right\}$,

where $L_{K}$ is the Kuhn step length and Z in Eq. 7 is not the drag tensor, but a normalization constant for the probability distribution. For DNA, we employ the worm-like chain spring law
$[23,24]$ for the stretch force:

$F^{\mathrm{str}}(s)=\frac{k_{B} T}{L_{K}}\left\{\left(1-\frac{s}{L}\right)^{-2}-1+4 \frac{s}{L}\right\}$

Now, the average drag tensor respectively $\langle\mathbf{Z}\rangle$ can be calculated as follows (using $\left.\zeta_{\perp}=2 \zeta_{\|}\right)$:

$$
\begin{aligned}
\langle\mathbf{Z}\rangle & =\zeta_{\|}\langle\mathbf{u u}\rangle+\zeta_{\perp}(\mathbf{I}-\langle\mathbf{u u}\rangle) \\
& =\zeta_{\|}(2 \mathrm{I}-\langle\mathbf{u u}\rangle) \\
& =\zeta_{\|}\left(\begin{array}{ccc}
2-\left\langle\cos ^{2} \theta\right\rangle & 0 & 0 \\
0 & 2-\left\langle\sin ^{2} \theta \cos ^{2} \phi\right\rangle & 0 \\
0 & 0 & 2-\left\langle\sin ^{2} \theta \sin ^{2} \phi\right\rangle
\end{array}\right),
\end{aligned}
$$

Using the EP velocity of a shish-kebab in Eq. (2), the term $\left\langle\mathbf{Z} \cdot \mathbf{v}^{\mathrm{SK}}\right\rangle$ is evaluated as,

$$
\begin{aligned}
\left\langle\mathbf{Z} \cdot \mathbf{v}^{\mathrm{SK}}\right\rangle_{x}= & \left\langle\zeta _ { \| } \left( v_{x}^{\mathrm{SK}} \cos ^{2} \theta+v_{y}^{\mathrm{SK}} \cos \theta \sin \theta \cos \phi\right.\right. \\
& \left.+v_{z}^{\mathrm{SK}} \cos \theta \sin \theta \sin \phi\right) \\
& +\zeta_{\perp}\left\{v_{x}^{\mathrm{SK}}\left(1-\cos ^{2} \theta\right)-v_{y}^{\mathrm{SK}}\right.
\end{aligned}
$$

$\left.\left.\cos \theta \sin \theta \cos \phi-v_{z}^{\mathrm{SK}} \cos \theta \sin \theta \sin \phi\right\}\right\rangle$

$$
=\zeta_{\|}\left\langle v_{x}^{\mathrm{SK}}\left(2-\cos ^{2} \theta\right)-v_{y}^{\mathrm{SK}}\right.
$$

$\left.\cos \theta \sin \theta \cos \phi-v_{z}^{\mathrm{SK}} \cos \theta \sin \theta \sin \phi\right\rangle$

$$
\begin{aligned}
& =\left\langle\left\{\mu_{0} E+\mu_{1} E\left(3 \cos ^{2} \theta-1\right)\right\}\left(2-\cos ^{2} \theta\right)\right\rangle \zeta_{\|} \\
& -\mu_{1} E\left\langle 3 \cos ^{2} \theta \sin ^{2} \theta \cos ^{2} \phi\right\rangle \zeta_{\|} \\
& -\mu_{1} E\left\langle 3 \cos ^{2} \theta \sin ^{2} \theta \sin ^{2} \phi\right\rangle \zeta_{\|} \\
& \left\langle\mathbf{Z} \cdot \mathbf{v}^{\mathrm{SK}}\right\rangle_{Y}=0 \\
& \left\langle\mathbf{Z} \cdot \mathbf{v}^{\mathrm{SK}}\right\rangle_{z}=0
\end{aligned}
$$

From the relation $\langle\mathbf{Z}\rangle \cdot \mathbf{V}=\left\langle\mathbf{Z} \cdot \mathbf{v}^{\mathrm{SK}}\right\rangle$, we find

$$
\begin{gathered}
V_{x}=\mu_{0} E+\mu_{1} E \\
\left\{\frac{\left\langle\left(3 \cos ^{2} \theta-1\right)\left(2-\cos ^{2} \theta\right)\right\rangle}{\left\langle 2-\cos ^{2} \theta\right\rangle}-2 \frac{\left\langle\frac{3}{2} \cos ^{2} \theta \sin ^{2} \theta\right\rangle}{\left\langle 2-\cos ^{2} \theta\right\rangle}\right\} \\
=\left[\mu_{0}+2 \mu_{1} h\left\{F^{\mathrm{str}}(s) L_{K} / k_{B} T\right\}\right] E, V_{Y}=0, V_{z}=0,
\end{gathered}
$$

where the function $h(x)$ is defined as $h(x)=-2+3 x^{2} /\left\{-2+x^{2}\right.$ $+2 x \operatorname{coth}(x)\}$. The function $h(x)$ monotonically increases from -0.2 to 1 when $x$ varies from 0 to infinity.

If we define $\mu_{\mathrm{EP}, \mathrm{HI}}$ to be the EP mobility with the effect of electrohydrodynamic interactions included, we can conclude from Eq. (11) that

$\mu_{\mathrm{EP}, \mathrm{HI}}=\mu_{0}+2 \mu_{1} h\left\{F^{\mathrm{str}}(s) L_{K} / k_{B} T\right\}$.

Since $h(x)$ is negative when $0<x<2.4, \mu_{\mathrm{EP}, \mathrm{HI}}$ becomes smaller than $\mu_{0}$ when the stretch of a sub-chain is small. This occurs because for small stretch there are many shishkebabs oriented perpendicular to the field, and these have a reduced contribution to the EP mobility relative to the mobility $\mu_{0}$ of isolated spheres (see Eq.(2)), and also have 
larger weighting factors for the overall center-of-mass velocity of a sub-chain than ones parallel to the field. From Eq. (12), and the fact that $h(x)$ varies between -0.2 and 1 as the chain is stretched, we find that the EP mobility of a highly stretched sub-chain can be enhanced by $66 \%$ over the EP mobility of a coiled (or unstretched) sub-chain when $\mu_{1}=\mu_{0} / 4$, which should hold when $\kappa a \leq 1$. Note that the hydrodynamic interaction from the elastic forces under uniform stretch of the sub-chain can be ignored because they do not induce any center-of-mass migration of the sub-chain.

With this expression for the mobility of each sub-chain, the dynamics of the full polymer chain, which is the fourth and final level of the hierarchical description, can be written down. This can include the effects of Brownian motion, which would allow one to simulate the detailed stretching dynamics of ensembles of chains electrophoresing in a nonuniform field (see Appendix A). Here, however, we are only interested in estimating the stretch dependence of the mobility, and in comparing this estimate with our experimental results. To keep the estimate simple, we will therefore assume that the stretch and the field $\mathbf{E}$ are uniform along the whole chain (not just the sub-chain), and use Eq. (12) to estimate the stretch dependence of the mobility. In reality, since it is a gradient in the electric field that stretches the chain, the chain stretch is not expected to be completely uniform along the molecule. Accounting for this non-uniformity is beyond the scope of this paper, however. By examining the predicted field gradient in the channel, we find that even when the molecule is stretched to the largest degree observed here (around $14 \mu \mathrm{m}$ ), the electric field varies by only around $18 \%$ over the length of the molecule. This modest variation in field helps justify our neglect of the non-uniformity of the stretch and our calculation of the mobility of the molecule using the magnitude of the field at the center of the molecule.

We note that a related approach to inclusion of electrohydrodynamic interactions has been presented by Butler et al. [25]. Their approach was directed towards explaining the electric-field-induced migration of DNA chains towards or away from the walls of thin channels, rather than towards the stretching phenomenon considered here. Their equations, while similar to ours (including the use of our Eq. (1) for the electrohydrodynamic disturbance field), were applied globally to a dumbbell model of the polymer, rather than locally to small segments within a single Kuhn length of the chain, as we do here. It is noteworthy that the paper of Butler et al. addressed experiments conducted at very low salt concentrations (much lower than the $10 \mathrm{mM}$ used in our experiments). Since we choose $\mu_{0}$ and $\mu_{1}$ for a shishkebab to be consistent with the ratio of the EP mobilities of an infinite cylinder oriented parallel to the field and perpendicular to the field, which holds over a wide range of salt concentration $(\kappa a \leq 1)$, our model should be applicable to salt concentrations below millimolar, as well. However, if the salt concentration becomes so low that the Debye length becomes larger than the Kuhn step length $L_{K}$, then the electrohydrodynamic interactions between shish-kebabs becomes non-negligible, and our modeling approach, which neglects hydrodynamic interactions between shish-kebabs, is no longer appropriate. For DNA $\left(L_{K} \cong 100 \mathrm{~nm}\right), \kappa L_{K}$ becomes order of one when the salt concentration is reduced to $10 \mu \mathrm{M}$.

\section{Results and discussion}

\subsection{Experimental results}

Since DNA is negatively charged, the DNA EP mobility is by definition negative. To analyze our experimental data, we first divide all EP mobility data into two groups based on the "visual length" of DNA, which is the length observed by microscopy and represents the degree of molecular stretch. The visual length of unstretched coiled DNA we observed in the experiment is less than around $3 \mu \mathrm{m}$. Therefore, we first choose the cutoff of $3 \mu \mathrm{m}$ to classify DNA into "unstretched" and "stretched". We present a histogram of EP mobilities for these two groups in Fig. 3A. The percentage of chains within each mobility bin is given by the height of the corresponding bar. It is clear that the EP mobility increases, on average, when the DNA is stretched. Although the range of DNA mobilities spans from -0.05 to $-0.37\left(10^{-8} \mathrm{~m}^{2} / \mathrm{sV}\right)$, the average values in the stretched and unstretched states are $-0.226 \pm 0.060$ and $-0.147 \pm 0.055$, respectively. We next scatter plot the EP mobilities versus the visual extended DNA length for all DNA data in Fig. 3B. (Note that the magnitude of the EP mobility increases downwards along the ordinate of Fig. 3B, because it is negative in sign.) The EP mobility is clearly not monotonic with respect to the visual length in the coil-stretch transition.

There is a large scatter in the data in Fig. 3B that can be partially reduced by correlating EP mobility with molecular conformation. In a hydrodynamically driven elongational flow, Perkins et al. [26] observed seven different DNA conformations: coiled, dumbbell, half-dumbbell, folded, uniform, kinked, and extended. In our study, five similar, but not necessarily identical, conformation types are found in the converging EP flow. We therefore classify our EP mobility data into five groups according to their conformations: 1- coiled, 2- oval, 3- folded, 4- half-dumbbell, and 5- extended, with individual snapshots illustrating these conformations in Fig. 4. Because of resolution limits, the folded conformation is not clearly distinguishable from a partially extended conformation. Some of transitions between these conformations observed in the experiments are given in the Supporting Information material. Based on this categorization, we examine the relationships between the conformation transition and the EP mobility. A slight elongation transforms a DNA conformation from a coiled sphere to an oval shape. Further elongation can unwind DNA into folded, half-dumbbell, and finally extended shapes. The EP mobility depends strongly on the instantaneous DNA conformation. According to Fig. 4, DNA with 

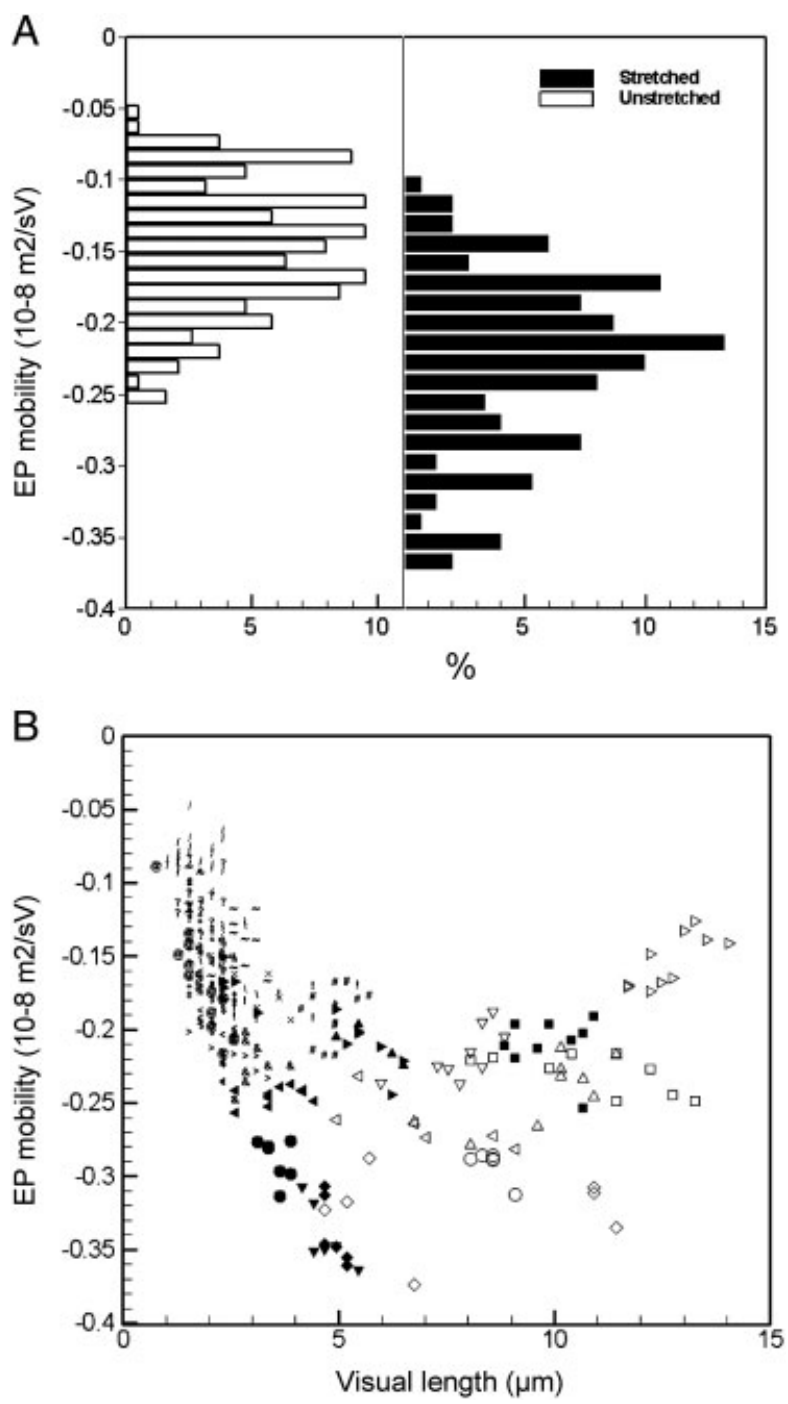

Figure 3. (A) Histogram of EP mobility for stretched and unstretched DNA. (B) Distribution of EP mobility versus DNA visual length. Each symbol type represents one tracked DNA molecule.

an oval shape has a much higher mobility than a coiled one of the same visual length. With increased visual length, the folded DNA attains a higher mobility magnitude than does the half-dumbbell or the extended ones.

In addition to the five conformations identified, we also highlight four EP mobility variation patterns in Fig 4. Three dashed lines track typical progressions of increased visual length from the coiled shape to explain the EP mobility variation owing to a conformation transition: (i) from coiled to oval, (ii) from coiled to folded, and (iii) from coiled to folded to half-dumbbell and then extended. The change of EP mobility depends on these conformational transitions as shown in Fig. 4. The EP mobility varies the most in pattern (i), followed by pattern (ii) and then pattern (iii). Therefore, "molecular individualism" affects not only the conformation transition in the coil-stretch process but also the EP mobility variation in electrophoresis. The fourth pattern (iv)

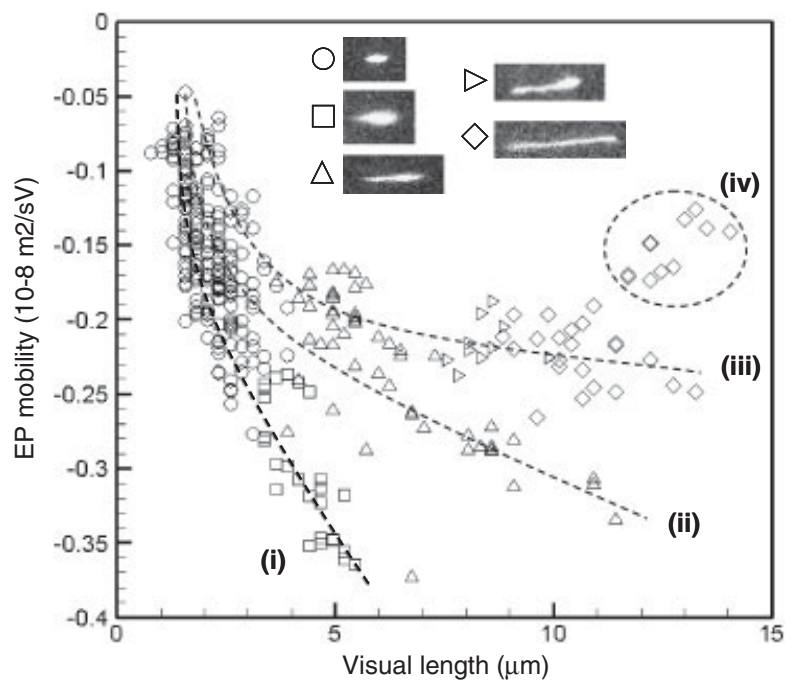

Figure 4. Distribution of EP mobility versus visual length for five DNA conformations. The fully stretched length of the DNA is around $20 \mu \mathrm{m}$. Different symbols correspond to DNA shapes depicted in the figure legend. (Circle: coiled, square: oval, triangle pointed upward: folded, rotated triangle pointed to the right: half-dumbbell, and diamond: extended). The three dashed lines mark three different unraveling paths typically taken by the molecules. The circled points correspond to "wiggling" conformations; see Section 3 in the text.

highlighted by the dashed circle is a "chain wiggling" phenomenon observed in extended DNA molecules. A movie showing this is provided in the Supporting Information material. The wiggling motion leads to a lower EP mobility, presumably because of higher energy dissipation.

\subsection{Theoretical results}

\subsubsection{EP mobilities of linear, U-shaped, and W-shaped stretch DNA}

Since we assume that all shish-kebab segments have the same ensemble average orientation for a linearly stretched DNA, our theory does not apply directly to conformations with nonuniform stretch such as dumbbell, half-dumbbell, or partially folded shapes. However, we still can calculate the mobility as a function of stretch ratio for evenly folded DNA chains such as a DNA with a U-shaped or a W-shaped stretch. If a U-shaped DNA at the steady state, which has a sharp bend at the middle of the chain, is cut at the middle into two pieces, then both pieces should migrate without changing their stretches at the same speed as the original U-shaped DNA, since the two pieces maintain the same force distribution in every section as the original. The only difference in mobility between a linearly stretched and a U-shaped molecule should then be the contour length. Therefore, the EP mobility of a U-shaped DNA is calculated simply by cutting the maximum stretch $L$ down to $L / 2$ in Eq. (8). Similarly, the EP mobility of a W-shaped DNA is calculated by cutting the maximum stretch down to $L / 4$. If we assume that the linearly stretch, U-shaped, and W-shaped 


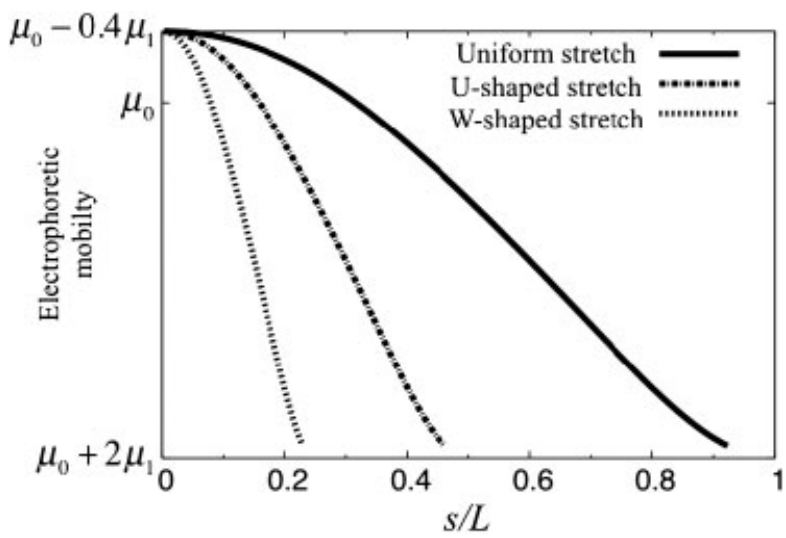

Figure 5. Theoretical estimation of the enhancement of the DNA EP mobility by stretching. Note that both $\mu_{0}$ and $\mu_{1}$ are negative for DNA. We expect $\mu_{1}=\mu_{0} / 4$ when $\kappa a \leq 1$.

conformations in the modeling can be interpreted, respectively, as the extended, folded, and oval shapes observed in the experiments, we can compare the results of experiment and modeling. Figure 5 shows the enhancement of the EP mobility for linear, U-shaped, and W-shaped stretch based on our theory, whose trend agrees well with the experimental result in Fig. 4 . Note that both $\mu_{0}$ and $\mu_{1}$ are negative for DNA. We also find that a more condensed structure (W and U-shape) has a higher mobility than does the straight stretch shape, again in agreement with our experimental observations.

\subsubsection{Comparison with the experiment}

Since the Debye thickness $\kappa^{-1}$ is around the range of $1-3 \mathrm{~nm}$ [8], which is comparable to the local radius of DNA chain, in our experimental condition, $\kappa a$ is order of one. Therefore, we expect $\mu_{1}=\mu_{0} / 4$ and the EP mobility of a highly stretched DNA is enhanced by $66 \%$ from the EP mobility of an unstretched DNA. By choosing $\left(\mu_{0}, \mu_{1}\right)=(0.2,0.05) \times 10^{-8}$ $\mathrm{m}^{2} / \mathrm{sV}$, the EP mobilities of unstretched and fully stretched DNA become $0.18 \times 10^{-8} \mathrm{~m}^{2} / \mathrm{sV}$ and $0.30 \times 10^{-8} \mathrm{~m}^{2} / \mathrm{sV}$, respectively, with $66 \%$ enhancement. Also, the calculated EP mobility time dependence for W- and U-shaped molecules are close to the experimental EP mobility patterns (i) and (ii), respectively, in Fig. 4, if we interpret the "oval" experimental configuration to be a "W" shape and the "folded" configuration to be a "U" shape. The calculated EP mobility profile of the linearly stretched molecule, however, does not agree very well with pattern (iii) because the actual conformational transition in pattern (iii) is more complicated than the simple linear stretch, and "chain wiggling" is not considered in the model.

\section{Concluding remarks}

DNA mobility in free solution is usually assumed to be a constant because DNA remains unstretched in a coiled configuration in free solution if the electric field is uniform. In this study, however, we have investigated theoretically and experimentally the effect of electrohydrodynamic interactions on $\lambda$-DNA EP mobility in free solution in a microscale converging channel, which produces conformational changes that induce changes in DNA conformation and hence in its mobility. DNA molecules were stretched by the electric field gradient in the converging section, and the resulting conformations were observed and instantaneous DNA EP mobility was calculated. We found that stretching increased the EP mobility and the change of EP mobility was conformation dependent. A shish-kebab model was proposed for each charged rigid charged "Kuhn step" of the DNA molecules and the shish-kebabs were linked into a freely jointed chain model for the flexible DNA polymer. We ensemble averaged the orientations of each shish-kebab rod using a field-dependent Boltzmann distribution and the wormlike-chain spring law to relate the stretching force on the shishkebabs to the overall chain stretch. Our modeling analysis shows that electrohydrodynamic interactions can enhance the EP mobility by stretching the DNA chains in a conformationdependent manner. The results are qualitatively consistent with the experimental observations, and show that EP mobility changes in a history-dependent manner, depending on whether the DNA unravels in a linear shape, or in a " $U$ " or "W" shape. These findings should be useful in interpreting and manipulating DNA mobility in complex geometries, including those used in biotechnological applications, such as gene delivery or DNA sequencing. In addition, we have presented equations for a more precise bead-spring model for DNA stretching and mobility in a non-uniform electric field.

The authors acknowledge support from NSF under grant NSEC EEC-0425626. They thank Patrick Doyle for helpful comments and for the Institute for Mathematics and Its Applications for support of RGL during its recent conference on Complex Fluids.

The authors have declared no conflict of interest.

\section{References}

[1] Deutsch, J. M., Science 1988, 240, 922-924.

[2] Randall, G. C., Doyle, P. S., Phys. Rev. Lett. 2004, 93, 058102.

[3] Juang, Y. J., Wang, S., Hu, X., Lee, L. J., Phys. Rev. Lett. 2004, 93, 268105.

[4] Gehl, J., Acta Physiol. Scand. 2003, 177, 437-447.

[5] Volkmuth, W. D., Austin, R. H., Nature 1992, 358, 600-602.

[6] Han, J., Craighead, H. G., Science 2000, 288, 1026-1029.

[7] Bader, J. S., Hammond, R. W., Henck, S. A., Deem, M. W., McDermott, G. A., Bustillo, J. M., Simpson, J. W., Mulhern, G. T. and Rothberg, J. M., Proc. Natl. Acad. Sci. U. S. A. 1999, 96, 13165-13169.

[8] Viovy, J. L., Rev. Mod. Phys. 2000, 72, 813-872.

[9] Long, D., Ajdari, A., Euro. Phys. J. E 2001, 4, 29-32. 
[10] Usta, O. B., Butler, J. E., Ladd, A. J. C., Phys. Rev. Lett. 2007, 98, 098301.

[11] Zheng, J. J., Yeung, E. S., Anal. Chem. 2002, 74, $4536-4547$.

[12] Zheng, J. J., Yeung, E. S., Anal. Chem. 2003, 75, 3675-3680.

[13] Landers, J. P., Handbook of Capillary Electrophoresis, CRC Press, Boca Raton 1997.

[14] Takagi, T., Electrophoresis 1993, 14, 1255-1256.

[15] Lindken, R., Rossi, M., Grosse, S., Westerweel, J., Lab Chip 2009, 9, 2551-2567.

[16] Hu, X., Wang, S. N., Lee, L. J., Phys. Rev. E 2009, 79, 12.

[17] deGennes, P. G., Science 1997, 276, 1999-1999.

[18] Perkins, T. T., Smith, D. E., Larson, R. G., Chu, S., Science 1995, 268, 83-87.

[19] Smith, D. E., Perkins, T. T., Chu, S., Macromolecules 1996, 29, 1372-1373.

[20] Russel, W. B., Saville, D. A., Schowalter, W. R., Colloidal Dispersions, Cambridge University Press, Cambridge, New York 1989.

[21] Yoon, B. J., Kim, S., J. Colloid Interface Sci. 1989, 128, 275-288.

[22] Doi, M., Edwards, S. F., The Theory of Polymer Dynamics, 1986.

[23] Bustamante, C., Marko, J. F., Siggia, E. D., Smith, S., Science 1994, 265, 1599-1600.

[24] Marko, J. F., Siggia, E. D., Macromolecules 1995, 28, 8759-8770.

[25] Butler, J. E., Usta, O. B., Kekre, R., Ladd, A. J. C., Phys. Fluids 2007, 19, 113101.

[26] Perkins, T. T., Smith, D. E., Chu, S., Science 1997, 276, 2016-2021.

\section{Appendix}

\section{Brownian dynamics simulations}

The dynamics of a charged polymer chain can be computed using a Brownian dynamics method by connecting sub-chains with stretch-dependent EP mobility $\mu_{E P, H I}$ in Eq. (12), where now the field $\mathbf{E}$ varies from one sub-chain to the next. By modeling a polymer with beads with hydrodynamic drag and springs that connect beads and represent sub-chains (i.e. the bead-spring model), the dynamics of the full chain can be computed according to the following equation of motion of each bead including the Brownian motion:

$\mathbf{r}_{\mathbf{i}}(\mathbf{t}+\delta \mathbf{t})=\mathbf{r}_{\mathbf{i}}(\mathbf{t})+\left\{\sum_{j=1}^{\mathrm{N}_{\mathrm{b}}} \frac{\mathbf{H}_{\mathrm{ij}} \cdot \mathbf{f}_{\mathbf{j}}}{\mathbf{k}_{\mathrm{B}} \mathbf{T}}+\mu_{\mathrm{i}} \mathbf{E}\left(\mathbf{r}_{\mathbf{i})}\right\} \delta \mathbf{t}+\sqrt{\mathbf{6} \delta \mathbf{t}} \sum_{\mathrm{j}=1}^{\mathrm{i}} \mathbf{B}_{\mathrm{ij}} \mathbf{n}_{\mathbf{i}}\right.$

where $\mathbf{r}_{i}(t)$ is the position of a bead $i$ at time $t, \mathbf{H}_{i j}\left(=\mathbf{B}_{i j} \cdot \mathbf{B}_{i j}^{\mathrm{T}}\right)$ is the Rotne-Prager-Yamakawa (RPY) hydrodynamic interaction tensor, $\mathbf{f}_{j}$ is the spring force on bead $j, N_{b}$ is the total number of beads in the polymer, $\mu_{i}$ is here the average EP mobility of the sub-chains adjacent to bead $i, \delta t$ is the time increment in the simulation, $\mathbf{n}_{j}$ is a random vector whose components are chosen from the range $[-1,1]$ in each time step, and $\mathbf{E}\left(\mathbf{r}_{i}\right)$ is the local electric field at bead $i$. With the above Eq. (A1), it would be possible to simulate an ensemble of polyelectrolyte chains traversing a tapered channel. Results could then be binned together depending on stretch and conformation type (such as "U" or "W" conformations) and the average mobility for each bin could be computed and compared with experimental values. Given the limitations of the data reported here, however, such detailed simulations are beyond the scope of this work. 